\title{
Optimization of the Response of Magnetoresistive Elements
}

\author{
KEES J. M. EIJKEL AND JAN H. J. FLUITMAN
}

\begin{abstract}
This paper provides a way to optimize the output signal of a general thin-film magnetoresistive element with a homogeneous magnetization field as used in applications with a saturating external magnetic field. The element is assumed to be operated by four-point measurement. In order to be able to compare different elements, a figure of merit is defined. The general theory of the anisotropic magnetoresistance effect (AMR effect) is treated. With that, a few general rules for optimization are formulated. It is concluded that in order to obtain a maximum signal voltage amplitude, the current density in the element should be constant, i.e., not affected by the AMR effect. It is shown, that the AMR effect on the current density in the element usually cannot be neglected. Some special configurations of magnetoresistive elements are treated in detail. The problem of four point contacts in an infinitely wide thin film is solved analytically, with the aid of a special transformation. It is found, that there is an optimum thickness of the thin film in an AMR device, which depends on the materia and the deposition technique. For pseudo-Hall elements, an optimum length-to-width ratio is found $(\approx 1.35)$.
\end{abstract}

\section{INTRODUCTION}

$\mathrm{D}$ URING the past few decades, the anisotropic magnetoresistance effect (AMR effect) in ferromagnetic thin films has been used to detect (variations of) magnetic fields in many applications [1], [2], etc. The signal voltage amplitude of AMR devices is usually rather small and amplification is required in most applications. If the noise from the AMR device is small, as usually is the case in applications where saturating external magnetic fields are used [2], the noise of the amplifier will be decisive for the signal-to-noise ratio of the system. In that case, an optimization of the signal voltage amplitude of the device is required. The resistor noise of AMR devices usually is negligible.

Optimization of AMR devices can be performed by optimizing the AMR material [3]-[5] or by optimizing the device geometry and the readout technique [6]-[8]. This can be done using an experimental approach [6], or with the aid of a theoretical model. However, these studies are usually concerned with special geometries of AMR devices. The optimization of a general device is very complicated. Furthermore, it depends on the actual detection problem in which the AMR effect is to be applied.

In this paper, the optimization concerns elements with a homogeneous magnetization, experiencing a saturating

Manuscript received February 9, 1989; revised August 11, 1989. This work was supported by the Netherlands Technology Foundation (STW).

The authors are with the Transducers and Materials Science Group, Faculty of Electrical Engineering, University of Twente, P.O. Box 217, 7500 AE Enschede, The Netherlands.

IEEE Log Number 8931783 in-plane magnetic field. The results of this study were used in the development of a contactless angle detector [2], [9].

A few general remarks will be made in advance:

- With the exception of a few special applications, the signal voltage amplitude of an AMR device should be stable in time and over a certain specified temperature range. This imposes constraints on the measurement setup: The contact resistance of the electrical contacts to the AMR film can be unstable at higher temperatures and current densities. On the other hand, the temperature behavior of the contact resistance can differ from that of the AMR film. Therefore, the influence of the contact resistance on the signal voltage of an AMR device should be minimized. This is achieved by driving the device with a highimpedance source (current source) and reading the signal voltage with a high-impedance amplifier (a four-point measurement setup). Such a measurement setup is assumed in the following calculations. A second problem can arise if a low-impedance driving and readout circuit is used: due to the magnetization-dependent internal resistance of most AMR devices, a distortion of the signal voltage can occur, which should be avoided in some applications [9].

- In all calculations the magnetization inside the AMR film is assumed to be in-plane. In practically all situations this assumption is valid, since the demagnetizing factor perpendicular to the film plane approaches 1 and because of the high saturation magnetization $M_{s}$ of the commonly used AMR materials.

- The magnetization in the film is assumed to be timeindependent and homogeneous. Its orientation will be given with respect to a reference axis, which is defined as the symmetry axis of the AMR device through the current contacts. If such a symmetry axis is absent in a device, another definition of the reference axis should be used. If the magnetization is time-dependent, eddy currents can occur in the film, giving rise to changes in the signal voltage. These eddy currents can usually be neglected if the magnetic field is in-plane. If inhomogeneities in the magnetization field of an AMR film are present, the response of the device should be examined combining magnetic and electrical properties. This can lead to different conclusions than presented here. As an example, the magnetization in the device may be influenced only at the edge of the film. In that case, the sensitivity of the device should be optimized in that area, e.g., by forcing the current to 
flow especially in that area. However, many of the conclusions drawn here can be applied to these devices.

- The influence of the current density on the magnetization in the film is extremely small and is neglected in the calculations.

- Only the AMR effect will be considered in our discussion. Other possible sources of resistivity anisotropy will be neglected. The most obvious source of resistivity anisotropy in a conductor is crystal anisotropy, which is canceled out in a polycrystalline film such as Permalloy. Furthermore, galvanomagnetic effects other than the AMR effect will be neglected. It should be noted, that the Hall effect in AMR films is so small as to be negligible [1].

- The magnetoresistive properties of the AMR film are assumed to be constant throughout the film plane.

This paper deals with the general theory of the current density and the electrical field in an AMR film with lowresistance contacts. Some conclusions can be drawn from this general theory. After that, a few special cases will be treated, leading to optimization criteria for the general device.

\section{The AMR MATERIAL}

The most commonly used AMR material is Permalloy, usually in the magnetostriction-free composition of about 81 at $\% \mathrm{Ni}$ and 19 at \% Fe. The ratio $81 / 19$ is close to that with the highest magnetoresistive effect which is around 90 at \% $\mathrm{Ni}[3]$. At the magnetostriction-free composition the magnetic properties of the Permalloy are superior: a very low coercive field of about $100 \mathrm{~A} / \mathrm{m}$ or lower for these very thin films and a low uniaxial anisotropy energy constant of about $200 \mathrm{~J} / \mathrm{m}^{3}$. These values are practically equal for films deposited by sputtering or by evaporation. Higher magnetoresistive effects are found in NiCo alloys [3] and NiFeCo alloys [4]. However, gains due to a higher magnetoresistivity are offset by a higher anisotropy constant and coercive field in these materials.

Optimization of the AMR material itself by optimizing the deposition technique is often possible. It should be mentioned, that special treatment of an AMR film can result in better performance. It is well known, for instance, that annealing treatment of Permalloy thin films lowers their resistivity by up to 30 percent, thus increasing the relative amplitude of the AMR effect itself [5], [9], [10], yielding higher output signals for a given input power.

\section{THE AMR EFFECT}

Fig. 1 shows an infinitely wide AMR film magnetized at an angle $\theta$ with the $x$ axis. Different resistivities $\rho_{\|}$and $\rho_{\perp}$ can be distinguished parallel and perpendicular to the magnetization vector $\left(\bar{M}_{s}\right)$, respectively. In this paper the amplitude of the AMR effect is defined as

$$
\Delta \rho=\frac{\rho_{\|}-\rho_{\perp}}{2}
$$

which is positive for most AMR materials.

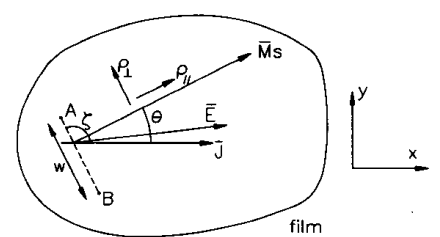

Fig. 1. A homogeneously saturated AMR film. The magnetization vector $\bar{M}_{s}$ is oriented at an angle $\theta$ with the $x$ axis. A homogeneous current density $\bar{J}$ is directed along the $x$ axis. Two point contacts $A$ and $B$ are positioned on the film surface at a mutual distance $w$.

The relative AMR effect (magnetoresistive ratio) is given by

$$
\frac{\Delta \rho}{\rho}=\frac{\rho_{\|}-\rho_{\perp}}{\rho_{\|}+\rho_{\perp}}
$$

with

$$
\rho=\frac{\rho_{\|}+\rho_{\perp}}{2}
$$

It should be noted that the definition of the AMR effect is slightly different from that, encountered in many other papers: $\Delta \rho=\rho_{\|}-\rho_{\perp}$. The definition used here is chosen because of the simpler form of the successive equations that will be presented. Definition (1) is used, among others, by Coren and Juretschke [11] and by Soohoo [12].

In a coordinate system with the $x$ and $y$ axes in the film plane, the $x$ axis parallel to the magnetization vector, the resistivity tensor of the film of Fig. 1 can be written as

$$
\overline{\bar{\rho}}=\left(\begin{array}{ccc}
\rho_{\|} & 0 & 0 \\
0 & \rho_{\perp} & 0 \\
0 & 0 & \rho_{z}
\end{array}\right)
$$

with $\rho_{z}$ the resistivity in the $z$ direction, perpendicular to the film plane. A homogeneous current density $\bar{J}$ is present in the film of Fig. 1, directed parallel to the $x$ axis. To determine the signal voltages that can be obtained from the film, we should first calculate the electric field $\bar{E}$.

When describing the behavior of an AMR device, it is more convenient to couple our coordinate system to the geometry of the device, or, equivalently, to the current direction in the film. In this case we choose the $x$ axis parallel to $\bar{J}$. Now $\overline{\bar{\rho}}$ will transform to $R \overline{\bar{\rho}} R^{-1}$ with $R$ the rotation in the $x-y$ plane over an angle $\theta$ around the origin

$$
R=\left(\begin{array}{ccc}
\cos \theta & -\sin \theta & 0 \\
\sin \theta & \cos \theta & 0 \\
0 & 0 & 1
\end{array}\right)
$$

We find

$$
\overline{\bar{\rho}}=\left(\begin{array}{ccc}
\rho_{\|}-2 \Delta \rho \sin ^{2} \theta & 2 \Delta \rho \sin \theta \cos \theta & 0 \\
2 \Delta \rho \sin \theta \cos \theta & \rho_{\|}-2 \Delta \rho \cos ^{2} \theta & 0 \\
0 & 0 & \rho_{z}
\end{array}\right)
$$


with $\Delta \rho$ as in (1). This can be written as

$$
\begin{aligned}
\overline{\bar{\rho}} & =\left(\begin{array}{ccc}
\rho+\Delta \rho \cos (2 \theta) & \Delta \rho \sin (2 \theta) & 0 \\
\Delta \rho \sin (2 \theta) & \rho-\Delta \rho \cos (2 \theta) & 0 \\
0 & 0 & \rho_{z}
\end{array}\right) \\
& =\left(\begin{array}{lll}
\rho_{x x} & \rho_{x y} & 0 \\
\rho_{x y} & \rho_{y y} & 0 \\
0 & 0 & \rho_{z}
\end{array}\right)
\end{aligned}
$$

with $\rho$ as in (3). From (5) it is obvious that the resistivity perpendicular to the film plane is independent of the (inplane) magnetization orientation. Because of the high ratio of in-plane dimensions to film thickness of thin-film AMR devices, the current density perpendicular to the film is zero everywhere, except in a negligibly small region around a current or voltage contact. From (5) it can be concluded, that the $E_{z}$ component of the $\bar{E}$ field will be zero throughout the film. This suggests, that we can reduce the problem to a two-dimensional one, simply by leaving out the $z$ component in the calculations. In a twodimensional approach the resistivity tensor reduces to

$$
\overline{\bar{\rho}}=\left(\begin{array}{ll}
\rho_{x x} & \rho_{x y} \\
\rho_{x y} & \rho_{y y}
\end{array}\right) .
$$

A two-dimensional approach can be used in many calculations concerning AMR devices. It should be noted, however, that the two-dimensional problem is not exactly analogous to the three-dimensional reality. By leaving out the $z$ component, the restriction that the current density is nonzero only inside the film $(0 \leq z \leq t$ with $t$ the film thickness ) is neglected. The three-dimensional analog of the two-dimensional approach is a pile of identical film planes extending to infinity along the $z$ axis. The $\bar{E}$ and $\bar{J}$ fields are independent of $z$. The two-dimensional solution is the cross section of this three-dimensional space with a plane $(z=$ constant $)$.

Using (5a), the $\bar{E}$ field at any point of the film of Fig. 1 can be calculated

$$
\bar{E}=\overline{\bar{\rho}} \bar{J}=\rho J\left(\begin{array}{l}
1 \\
0
\end{array}\right)+\Delta \rho J\left(\begin{array}{c}
\cos (2 \theta) \\
\sin (2 \theta)
\end{array}\right) .
$$

The first term on the right is the $\bar{E}$ field in an isotropic conductor with resistivity $\rho$. The second term is the AMR term, which depends on the magnetization direction in the film. It is interesting to see, that the modulus of the latter is $\Delta \rho J$, independent of the magnetization direction, while its orientation is coupled directly with the magnetization direction. It is clear from (6), that $\bar{J}$ and $\bar{E}$ will usually not be parallel in an AMR film.

In a practical situation, two contacts $A$ and $B$ will be positioned on the film at a mutual distance $w$, to measure a voltage difference $V_{\text {sig. }}$. It is presumed that these contacts do not affect the current density in the film; in other words, we have point contacts that convey no current. $V_{\text {sig }}$ can now be calculated by taking $\int \bar{E} \cdot d \bar{l}$ on the straight line connecting the voltage contacts. If this line is oriented at an angle $\zeta$ with $\bar{J}$, the component of $\bar{E}$ parallel to the line reads

$$
E_{\zeta}=\rho J \cos \zeta+\Delta \rho J \cos (2 \theta-\zeta) .
$$

In the present case, $E_{\zeta}$ is constant along this line, and $V_{\text {sig }}$ can be calculated

$$
\begin{aligned}
V_{\text {sig }} & =\int_{A}^{B} E_{\zeta} d l \\
& =\rho J w \cos \zeta+\Delta \rho J w \cos (2 \theta-\zeta) .
\end{aligned}
$$

By changing the value of $\zeta$, the phase of the AMR term in $V_{\text {sig }},(2 \theta-\zeta)$, can be adapted. The amplitude of the AMR effect remains constant as long as the distance between the voltage contacts is left unchanged.

Two well-known configurations using the AMR effect are the magnetoresistor and the planar Hall or pseudoHall device [1], shown in Fig. 2(a) and (b), respectively. The current density in the films will be homogeneous, so $J=I / w t$ with $I$ the driving current, $w$ the width, and $t$ the thickness of the Permalloy strip. The current contacts have a mutual distance of $l$. To reduce the influence of short-circuiting effects on the signal voltages, we take $l \gg w$.

In the magnetoresistor we have $(\zeta=0)$

$$
\begin{aligned}
V_{\mathrm{mr}} & =\rho J l+\Delta \rho J l \cos (2 \theta) \\
& =I \frac{\rho}{t} \frac{l}{w}+I \frac{\Delta \rho}{t} \frac{l}{w} \cos (2 \theta) \quad(l \gg w) .
\end{aligned}
$$

Usually, this voltage is written as

$$
V_{\mathrm{mr}}=I R-I \Delta R \sin ^{2} \theta
$$

with $R$ the resistance of the magnetoresistor taken at $\theta=$ 0 and $I$ the driving current of the device. $\Delta R$ is the maximum change in strip resistance for different magnetization directions. It is related to both the shape of the magnetoresistor and the (intrinsic) AMR effect. For the sake of clarity of the description, it is more convenient to separate material and geometry parameters, as in (9).

For the pseudo-Hall device we find $\left(\zeta=\frac{1}{2} \pi\right)$

$$
V_{\mathrm{ph}}=\Delta \rho J w \sin (2 \theta)=I \frac{\Delta \rho}{t} \sin (2 \theta) \quad(l \gg w) .
$$

Usually, this so-called pseudo-Hall voltage is written as

$$
V_{\mathrm{ph}}=\frac{w}{l} I \Delta R \sin \theta \cos \theta=\frac{1}{2} I \Delta R \frac{w}{l} \sin (2 \theta) .
$$

$\Delta R$ has the same definition as in the magnetoresistor, a combination of material and geometry parameters. The ratio $(w / l)$ of the device does not influence the pseudoHall voltage (apart from the short-circuiting effect, which is not dealt with here), since $\Delta R$ contains a factor $(l / w)$, 


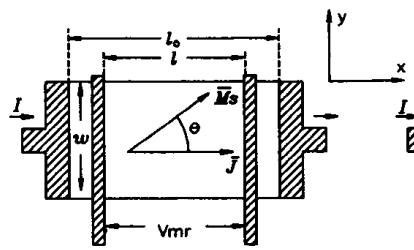

(a)

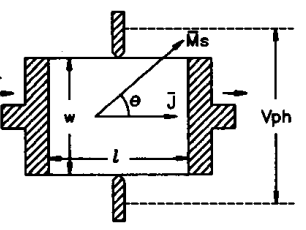

(b)
Fig. 2. (a) A magnetoresistor. (b) A pseudo-Hall element.

as can be seen in (10). To avoid this unclear situation and to use a single formalism for all AMR-related effects in the AMR devices, the formalism of (6) and (8) will be used throughout this paper. From this point of view, the magnetoresistive effect (9) and the pseudo-Hall effect (10) are special cases of the AMR effect (6). It is interesting to see, that in (9) and (10) the resistivity $\rho$ and AMR effect $\Delta \rho$ are divided by the thickness $t$, as in all equations encountered in the following. This is a consequence of the approximately two-dimensional geometry of thin-film devices.

In (9) and (10) the current density in the devices was considered to be independent of the magnetization direction in the AMR film. In general, this is only an approximation, since the low-resistance current contacts of the devices of Fig. 2 short-circuit the cross voltage that builds up over the strip width (the short-circuiting effect). Therefore, (9) and (10) are only valid for long strips $(l \gg w)$, where the influence of the short-circuiting effect on the signal voltage is negligible.

\section{The General Theory}

In general, the Permalloy film will not be infinite, but will have a certain in-plane geometry, while the contacts will have finite size. The current density is determined by these geometry parameters and will generally not be homogeneous. In this section, the two-dimensional problem will be solved using the three-dimensional analog of a pile of identical films extending to infinity in the $+z$ and $-z$ directions.

If the resistivity in the film is isotropic, the current density is independent of the magnetization in the film. Contact geometry and film geometry determine the current density in the film. In (6) this means, that $\bar{J}$ is not homogeneous, but dependent on the actual position: $\bar{J}=$ $\bar{J}(x, y)$. If a resistance anisotropy is introduced, the current density is generally affected. This means, that $\bar{J}$ will also depend on the orientation of the resistance anisotropy and consequently on the direction of $\bar{M}_{s}$, so $\bar{J}=\bar{J}(x, y$, $\theta)$. When calculating signal voltages from AMR devices, $\bar{J}$ is often thought to be independent of the AMR effect as illustrated in the previous section [1]. However, in a universal AMR device this will lead to erroneous results and a more general approach is needed to calculate $V_{\text {sig }}$.

In a point that is not a current source or sink, and under the condition $d \bar{B} / d t=0$ for external magnetic fields, we know that

$$
\operatorname{div} \bar{J}=0
$$

and

$$
\operatorname{curl} \bar{E}=0 \text {. }
$$

With $\operatorname{div} \bar{J}=0$ we can write: $\bar{J}=-\operatorname{curl} \bar{\Psi}$, the curl of a vector field. In our thin film with $J_{z}=0$ everywhere, we can state without loss of generality that

$$
\bar{J}=-\operatorname{curl} \bar{\Psi}=-\operatorname{curl}\left(\begin{array}{l}
0 \\
0 \\
\Psi
\end{array}\right)=\left(\begin{array}{c}
-\partial \Psi / \partial y \\
\partial \Psi / \partial x \\
0
\end{array}\right) .
$$

A line $\Psi=$ constant represents a current path: if $\Psi=$ constant along a curve in our AMR film defined by the points $(x(\xi), y(\xi), 0)$, we know that

$$
\frac{d \Psi}{d \xi}=\frac{\partial \Psi}{\partial x} \frac{d x}{d \xi}+\frac{\partial \Psi}{\partial y} \frac{d y}{d \xi}=0
$$

so

$$
\frac{d y}{d x}=\frac{\frac{d y}{d \xi}}{\frac{d x}{d \xi}}=\frac{\frac{\partial \Psi}{\partial x}}{-\frac{\partial \Psi}{\partial y}}=\frac{J_{y}}{J_{x}}
$$

so the tangent to the line $\Psi=$ constant has the direction of $\bar{J}$ at that point.

$\bar{E}$ can be written as $-\operatorname{grad} \Phi$, the gradient of a scalar field $\Phi(x, y)$ representing the potential at each point in the film.

$$
\bar{E}=-\operatorname{grad} \Phi=\left(\begin{array}{c}
-\partial \Phi / \partial x \\
-\partial \Phi / \partial y \\
0
\end{array}\right)
$$

with the $z$ derivative of $\Phi$ equal to zero.

With (12), (13), and $\bar{E}=\overline{\bar{\rho}} \bar{J}$ with $\overline{\bar{\rho}}$ as in (5), we find

$$
(\operatorname{curl} \bar{E})_{z}=\rho_{x x} \frac{\partial^{2} \Psi}{\partial y^{2}}-2 \rho_{x y} \frac{\partial^{2} \Psi}{\partial x \partial y}+\rho_{y y} \frac{\partial^{2} \Psi}{\partial x^{2}}=0 .
$$

At the edges of the film, the boundary conditions should be satisfied.

Presume the boundary of the film is described by the parameter equation

$$
\left.\begin{array}{l}
x=f(\xi) \\
y=g(\xi) \\
z=z
\end{array}\right\}
$$

If there is no (current or voltage) contact at the film edge, the edge is a current path, and we have

$$
\Psi(f(\xi), g(\xi), z)=\text { constant } .
$$


If a (highly conductive) contact is positioned at the film edge, we know that $\Phi=$ constant, so there is no component of $\bar{E}$ parallel to the contact boundary. The orientation of the boundary at any point along the contact can be calculated from (16), and we find

$$
(\overline{\bar{\rho}} \bar{J}) \cdot \bar{T}=0
$$

with $\bar{T}$ a vector in the plane of the AMR film, tangential to the contact boundary.

Equations (15), (17), and (18) lead to a solution for the current density in the film. $\bar{E}$ and $\Phi$ can be found with (6) and (14). When $\Phi$ is known, $V_{\text {sig }}$ can be calculated as the potential difference between the two voltage contacts. Note that the $z$ components in (17) and (18) can be left out again, reducing the three-dimensional equations to two-dimensional equations.

An analogous approach uses (11) with $\bar{J}$ replaced by $\overline{\bar{\sigma}} \bar{E}, \overline{\bar{\sigma}}$ being the conductivity tensor in the material

$$
\bar{J}=\overline{\bar{\sigma}} \bar{E}=\left(\begin{array}{ccc}
\sigma_{x x} & \sigma_{x y} & 0 \\
\sigma_{x y} & \sigma_{y y} & 0 \\
0 & 0 & \sigma_{z}
\end{array}\right) \bar{E} .
$$

The matrix coefficients $\sigma_{x x}, \sigma_{x y}$, and $\sigma_{y y}$ can be expressed in terms of the conductivity along $\bar{M}, \sigma_{\|}$, and the conductivity perpendicular to $\bar{M}, \sigma_{\perp}$, in the same way as with the calculation of the tensor coefficients of $\overline{\bar{\rho}}$ (as before, $\bar{M}$ is in the film plane). We have

$$
\begin{array}{cc}
\sigma_{\|}=1 / \rho_{\|} \quad \sigma=\left(\sigma_{\|}+\sigma_{\perp}\right) / 2 \\
\sigma_{\perp}=1 / \rho_{\perp} \quad \Delta \sigma=\left(\sigma_{\|}-\sigma_{\perp}\right) / 2 \\
\sigma_{z}=1 / \rho_{z} \\
\sigma_{x x}=\sigma+\Delta \sigma \cos (2 \theta) \\
\sigma_{x y}=\quad \Delta \sigma \sin (2 \theta) \\
\sigma_{y y}=\sigma-\Delta \sigma \cos (2 \theta) .
\end{array}
$$

Note that $\Delta \sigma$ has opposite sign as $\Delta \rho$. Combining (11), (19), and (14) we find a differential equation for $\Phi$

$$
\sigma_{x x} \frac{\partial^{2} \Phi}{\partial x^{2}}+2 \sigma_{x y} \frac{\partial^{2} \Phi}{\partial x \partial y}+\sigma_{y y} \frac{\partial^{2} \Phi}{\partial y^{2}}=0
$$

or

$$
\rho_{x x} \frac{\partial^{2} \Phi}{\partial y^{2}}-2 \rho_{x y} \frac{\partial^{2} \Phi}{\partial x \partial y}+\rho_{y y} \frac{\partial^{2} \Phi}{\partial x^{2}}=0 .
$$

The boundary equations for $\Phi$ are identical to those for $\Psi$, when the film boundaries and contact boundaries are interchanged.

With (20) and the boundary equations for $\Phi$, we can find $\Phi$ directly and calculate $V_{\text {sig }}$ by taking the difference between the values of $\Phi$ at the voltage contacts. It is interesting to see, that $\Phi$ and $\Psi$ satisfy the same differential equation with the boundary conditions for the film edge and for the contacts interchanged. This means that the equipotential lines in a film will coincide with the current paths in the film when the current contacts and film edges are interchanged.

The solutions to (15) or (20) cannot be calculated analytically for an arbitrary geometry. It is only for very simple structures that the solution can be found. A numerical analysis should be performed for a general geometry.

Before conclusions can be drawn from the equations derived above, we should first consider the following:

The angle $\alpha$ between the $\bar{E}$ field and the $\bar{J}$ field as a function of $\theta$ can be calculated from (6) [13]

$$
\begin{aligned}
\alpha & =\arctan \left(\frac{\frac{\Delta \rho}{\rho} \sin (2 \theta)}{1+\frac{\Delta \rho}{\rho} \cos (2 \theta)}\right) \\
& \approx \frac{\Delta \rho}{\rho} \sin (2 \theta), \quad \text { if } \frac{\Delta \rho}{\rho} \ll 1 .
\end{aligned}
$$

The maximum value of $\alpha$ only depends on the ratio $\Delta \rho / \rho$ and is about $1^{\circ}$ in Permalloy films. The AMR-induced changes in the $\bar{E}$ and $\bar{J}$ fields are divided between both fields. Depending on the boundary equations belonging to (15) or (20), one of these fields can be fixed so that the other shows maximum changes with varying magnetization direction. As an example, we take the magnetoresistor of Fig. 2(a). If $l \gg>$, the boundary equations dictate that the current density is homogeneous throughout the AMR film (except in a small region close to the contacts). If the device is driven by a constant current, the current density is independent of the magnetization direction. The electric field shows maximum AMR-induced changes described by (6). The signal voltage is given by (9).

On the other hand, if $l \ll w$, the boundary equations dictate that the $\bar{E}$ field is homogeneous throughout the AMR film, and if the device is driven by a constant voltage, the electric field is independent of the magnetization direction. The current density will now show maximum AMR-induced changes. With $\bar{J}=\overline{\bar{\sigma}} \bar{E}$, we find the total current $I_{\text {sig }}$ through the device to be

$$
I_{\text {sig }}=V \sigma t \frac{w}{l}+\dot{V} \Delta \sigma t \frac{w}{l} \cos (2 \theta)
$$

where $V$ is the constant voltage between the current contacts. Driving the device with constant voltage should be avoided, because of temperature-dependent contact resistances. In a practical situation one can use a constant current supply in this type of device. A signal voltage can be measured at the contacts. From (9a) one can see, that this signal voltage will resemble (9) with only a small distortion. An example of a device of this type is the barberpole element [14]. In fact, changes in the current density are measured by exploiting the resistivity of the material in this configuration.

In pseudo-Hall devices or their equivalents, the electric field should not be fixed. A measurement of $V_{\text {sig }}$ will not 
show an AMR effect in that case. In these elements, the current density should be fixed.

In order to obtain a fixed $\bar{J}$ field, we should examine (15) and the boundary equations for the device. Equation (15) is treated in the next section. Concerning the boundary equations, it can be concluded that in the case of a pseudo-Hall-like device the contacts should only have a minor influence on the current density in the region of the AMR film that determines $V_{\text {sig. }}$. In other words, the voltage contacts should be small, while the current contacts should be either small or far away from the voltage contacts $(l \gg w)$ to avoid the so-called short-circuiting effect.

\section{Current Density Fields Insensitive to the AMR EFFECT}

The signal voltage in a pseudo-Hall-like device is determined by the changes in the $\bar{E}$ field and will be largest when the $\bar{J}$ field is fixed. From that point of view, it is useful to examine which current densities are unchanged by the AMR effect in an AMR film.

The subsequent calculation of the signal voltage is relatively easy in that case: the $\bar{E}$ field can be calculated directly with (6) and $V_{\text {sig }}$ is obtained by integration.

In the case of an infinitely wide AMR thin film we can use (15) to find $\Psi$, which can be rewritten as

$$
\begin{gathered}
\rho \frac{\partial^{2} \Psi}{\partial x^{2}}+\rho \frac{\partial^{2} \Psi}{\partial y^{2}}+\Delta \rho \cos (2 \theta)\left(\frac{\partial^{2} \Psi}{\partial x^{2}}-\frac{\partial^{2} \Psi}{\partial y^{2}}\right) \\
-2 \Delta \rho \sin (2 \theta) \frac{\partial^{2} \Psi}{\partial x \partial y}=0 .
\end{gathered}
$$

If the last two terms equal zero, we get the desired independence of the solution of $\Psi$ from the direction of magnetization, represented by $\theta$. What remains is the solution for an isotropic conductor. To achieve this, it is necessary that

$$
\left(\frac{\partial^{2} \Psi}{\partial x^{2}}-\frac{\partial^{2} \Psi}{\partial y^{2}}\right)=0
$$

and

$$
\frac{\partial^{2} \Psi}{\partial x \partial y}=0 .
$$

This set of differential equations can be solved quite easily, leading to

$$
\bar{J}=a\left(\begin{array}{r}
y \\
-x
\end{array}\right)+\left(\begin{array}{l}
c_{1} \\
c_{2}
\end{array}\right)
$$

with $a, c_{1}$, and $c_{2}$ being arbitrary constants. Equation (21) is a superposition of a homogeneous current density and a circular current density with an amplitude growing linearly with the distance from its center. This circular current density can be an eddy current in a homogeneous alternating field perpendicular to the film plane. The radius of every circle increases linearly with the distance from the origin, while the flux through the circle increases

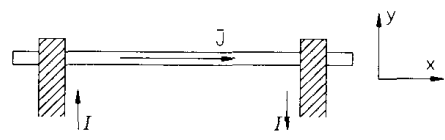

Fig. 3. Approximation of a homogeneous current density in a finite geometry. Only in the region close to the current contacts is the homogeneity of the $\bar{J}$ field violated.

quadratically, giving a circular current density as shown in the first right-hand term in (21). The use of eddy currents to obtain a driving current in an AMR film could be considered. However, in a real AMR device problems will arise when strong alternating fields are allowed near the sensor: the orientation of the magnetization will be affected and crosstalk will occur between the signal cables, to mention only two of these problems. Furthermore, it is a practical problem to build a thin-film coil that produces a sufficient field strength at a high enough frequency to allow operation of the sensor in this setup. It can be concluded that in practical situations in very large films only homogeneous current densities remain unchanged by the AMR effect.

In a finite film geometry, the boundary conditions impose extra demands on the current density. Far from the boundaries, the solution for $\bar{J}$ will resemble that of an infinite film, and only homogeneous current densities remain unchanged by the AMR effect. Therefore, the boundary equations should be satisfied by the solution of a homogeneous current density. This is realized in rectangular strips as in Fig. 3. The current flows parallel to the strip length. The boundary conditions at the sides of the strip are satisfied by a homogeneous current density along the strip length

$$
\bar{J}=\left(\begin{array}{l}
J_{x} \\
0
\end{array}\right)
$$

which is the solution for $\bar{J}$ for the total strip, apart from the regions close to the current contacts. At those regions, the current density depends on the direction of magnetization in the film (the short-circuiting effect).

The stability of homogeneous current densities in infinitely wide anisotropic films justifies the assumed stability of the current density in the infinite film of Fig. 1.

Another way to construct current densities unchanged by the AMR effect is to let the boundary equations (17) dominate.

\section{Point Contacts in an Infinitely Wide AMR Film}

To give an example of the influence of AMR-induced changes in the current density on the signal voltage of an AMR device, the geometry of Fig. 4 will be treated. Two point contacts are positioned on an infinite Permalloy thin film of thickness $t$. The contacts are placed in $(1,0)$ and in $(-1,0)$ and driven by a current $I$ which enters the film at $(1,0)$. Two extra point contacts can be positioned at arbitrary points on the film to measure a signal voltage, which can be calculated by solving (20) for this geometry. To find the solution, we will first look at an isotropic film. 


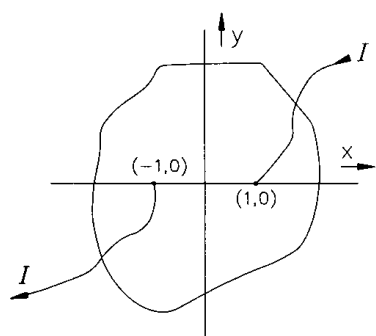

Fig. 4. An infinite Permalloy film with two point contacts.

There is a close similarity between the solution of the $\bar{E}$ field around a point charge $q$ and the $\bar{J}$ field around a point contact driven with current $I$ in an isotropic conductor. The form of the fields is the same, they differ only by a constant. Gauss's law tells us that the total electric flux through a closed surface around $q$ is $q / \epsilon_{0}$. The total current flux through a closed surface around a point contact is $I$. Therefore, the current density $\bar{J}$ around a point contact can be calculated in the same way as the electric field $\bar{E}$ around a point charge, when $q / \epsilon_{0}$ is replaced by $I$ in Coulomb's law. In an isotropic medium we have $\rho \vec{J}=\vec{E}$, so the electric field $\bar{E}$ around the point contact is found directly by replacing $q / \epsilon_{0}$ by $I \rho$, changing Coulomb's law into a form

$$
\bar{E}=\frac{I \rho}{4 \pi r^{2}} \bar{e}_{r}
$$

or

$$
\Phi=\frac{-I \rho}{4 \pi r}
$$

with $\bar{e}_{r}$ a unit vector from the point contact to the point where $\bar{E}$ is calculated, and $r$ the distance between these points. In a very thin film, with $\bar{J}$ independent of the position perpendicular to the plane, (22) changes into

$$
\bar{E}=\frac{I \rho}{2 \pi r t} \bar{e}_{r}
$$

or

$$
\Phi=\frac{-I \rho}{2 \pi t} \ln (r)
$$

with all vectors in the film plane.

In (23), $\Phi \rightarrow \infty$ for $r \rightarrow \infty$. This is caused by the fact, that we are really calculating the potential field for a line source of current in three-dimensional space, which goes to infinity for $r \rightarrow \infty$. The electric field of a set of two point contacts conveying currents $I$ and $-I$ at a distance $r_{1}$ and $r_{2}$, respectively, reads

$$
\bar{E}=\frac{I \rho \bar{e}_{r 1}}{2 \pi r_{1} t}-\frac{I \rho \bar{e}_{r 2}}{2 \pi r_{2} t}
$$

or

$$
\Phi=\frac{I \rho}{2 \pi t} \ln \left(\frac{r_{2}}{r_{1}}\right)
$$

If these point contacts are positioned at $(-1,0)$ and $(1,0)$ as in Fig. 4 , this leads to

$$
\Phi=\frac{I}{2 \pi} \frac{\rho}{t} \ln \left(\frac{\sqrt{(x+1)^{2}+y^{2}}}{\sqrt{(x-1)^{2}+y^{2}}}\right) .
$$

In this case, $\Phi$ goes to zero at infinity.

If we are dealing with an anisotropic resistivity, (24) will not be valid. However, by performing a suitable transformation of the anisotropic situation [15], we can translate the problem into an isotropic one, and (24) can be used directly to find the solution.

If the coordinate system is rotated over an angle $\theta$ to a new $x^{\prime}-y^{\prime}$ coordinate system, so that the $x^{\prime}$ axis coincides with the magnetization vector, the resistivity tensor will have a form as in (4) and the cross terms in (15) and (20) will vanish. Scaling of the axes $\left(\xi=\sqrt{\rho_{\|}} x^{\prime}\right.$ and $\eta=$ $\left.\sqrt{\rho_{\perp}} y^{\prime}\right)$ changes (15) and (20) into the Laplace equations $\nabla^{2} \Psi=0$ and $\nabla^{2} \Phi=0$. The total transformation has a matrix form

$$
T=\left(\begin{array}{cc}
\sqrt{\rho_{\|}} \cos \theta & \sqrt{\rho_{\|}} \sin \theta \\
-\sqrt{\rho_{\perp}} \sin \theta & \sqrt{\rho_{\perp}} \cos \theta
\end{array}\right) .
$$

Transformation of the geometry of Fig. 4 to the $\xi-\eta$ plane and application of (24) directly leads to a solution for $\Phi(\xi$, $\eta$ ). The resistivity in the $\xi-\eta$ plane can be determined as follows (see also (13) and (14)):

$$
\begin{aligned}
\bar{E}^{*} & =\left(\begin{array}{l}
-\partial \Phi / \partial \xi \\
-\partial \Phi / \partial \eta
\end{array}\right) \\
& =\left(\begin{array}{ll}
\partial x / \partial \xi & \partial y / \partial \xi \\
\partial x / \partial \eta & \partial y / \partial \eta
\end{array}\right)\left(\begin{array}{ll}
-\partial \Phi / \partial x \\
-\partial \Phi / \partial y
\end{array}\right) \\
& =\left(\begin{array}{ll}
\partial x / \partial \xi & \partial y / \partial \xi \\
\partial x / \partial \eta & \partial y / \partial \eta
\end{array}\right) \bar{E} \\
& =\left(\begin{array}{ll}
\partial x / \partial \xi & \partial y / \partial \xi \\
\partial x / \partial \eta & \partial y / \partial \eta
\end{array}\right) \overline{\bar{\rho}} \\
& =\left(\begin{array}{ll}
\partial x / \partial \xi & \partial y / \partial \xi \\
\partial x / \partial \eta & \partial y / \partial \eta
\end{array}\right) \overline{\bar{\rho}}\left(\begin{array}{rr}
\partial \eta / \partial y & -\partial \xi / \partial y \\
-\partial \eta / \partial x & \partial \xi / \partial x
\end{array}\right) \bar{J}^{*} \\
& =\overline{\bar{\rho}}^{*} \bar{J}^{*}
\end{aligned}
$$

in which $\bar{E}^{*}, \bar{J}^{*}$, and $\overline{\bar{\rho}} *$ are given in coordinates with respect to the $\xi-\eta$ axes. The resistivity tensor in the $\xi-\eta$ plane can now be calculated. The partial derivatives can be obtained from the definition of $T$ in (25) and the inverse transformation $T^{-1}$, leading to

$$
\overline{\bar{\rho}}^{*}=\left(\begin{array}{cc}
\sqrt{\rho_{\|} \rho_{\perp}} & 0 \\
0 & \sqrt{\rho_{\|} \rho_{\perp}}
\end{array}\right)=\left(\begin{array}{cc}
\rho^{*} & 0 \\
0 & \rho^{*}
\end{array}\right) .
$$

The resistivity in the $\xi-\eta$ plane is isotropic, as expected, with a value

$$
\rho^{*}=\sqrt{\rho_{\|} \rho_{\perp}}=\sqrt{(\rho+\Delta \rho)(\rho-\Delta \rho)}
$$


which is an alternative definition for average resistivity in the material. With this, (24) yields

$$
\begin{aligned}
\Phi(\xi, \eta) & =\frac{I}{2 \pi} \frac{\rho^{*}}{t} \\
& \cdot \ln \left(\frac{\sqrt{\left(\xi+\sqrt{\rho_{\|}} \cos \theta\right)^{2}+\left(\eta-\sqrt{\rho_{\perp}} \sin \theta\right)^{2}}}{\sqrt{\left(\xi-\sqrt{\rho_{\|}} \cos \theta\right)^{2}+\left(\eta+\sqrt{\rho_{\perp}} \sin \theta\right)^{2}}}\right)
\end{aligned}
$$

or

$$
\begin{aligned}
\Phi(x, y) & =\frac{I}{2 \pi} \frac{\rho^{*}}{t} \\
& \cdot \ln \left(\frac{\sqrt{\rho_{x x}(x+1)^{2}+\rho_{y y} y^{2}+2(x+1) y \rho_{x y}}}{\sqrt{\rho_{x x}(x-1)^{2}+\rho_{y y} y^{2}+2(x-1) y \rho_{x y}}}\right) .
\end{aligned}
$$

If two voltage contacts are positioned on the $x$ axis (magnetoresistor geometry) at a distance $w$, symmetrically around the origin, we find

$$
V_{\text {sig }}=\frac{I}{\pi} \frac{\rho^{*}}{t} \ln \left(\frac{|w+2|}{|w-2|}\right)
$$

with a singularity for $w=2$, when the voltage contacts coincide with the current contacts. $V_{\text {sig }}$ is independent of the magnetization direction. This effect was demonstrated experimentally in a circular film with a diameter of $5 \mathrm{~cm}$. The contacts were positioned as in Fig. 5(a). The variation in $V_{\text {sig }}$ with rotating magnetization was less than 0.25 percent, more than 10 times smaller than the signal voltage swing in a magnetoresistor. The remaining variation in $V_{\text {sig }}$ is attributed to the finite size of the contacts used.

If the voltage contacts are positioned on the $y$ axis at a distance $w$, symmetrically around the origin (pseudo-Hall geometry), we find

$$
V_{\text {sig }}=\frac{I}{2 \pi} \frac{\rho^{*}}{t} \ln \left(\frac{\rho_{x x}+\left(w^{2} / 4\right) \rho_{y y}+w p_{x y}}{\rho_{x x}+\left(w^{2} / 4\right) \rho_{y y}-w \rho_{x y}}\right) .
$$

For an AMR effect of a few percent or less (as in Permalloy) and $w=2,(29)$ is approximated by

$$
V_{\mathrm{sig}}=\frac{I}{\pi} \frac{\Delta \rho}{t} \sin (2 \theta) \text {. }
$$

This means, that the signal-voltage amplitude is reduced by a factor $\pi$ with respect to the pseudo-Hall configuration of Fig. 2(b). With $w=2$, only half the current passes between the voltage contacts. This accounts for a factor of 2 in the reduction of the signal voltage. A factor of $\pi / 2$ is caused by the fact that the current density is affected by the AMR effect. Changes in the current density occur at the cost of changes in the electric field, and thus at the cost of the signal-voltage amplitude.

In general, the signal voltage (29) will not have an exact sine shape as a function of $\theta$, in contrast with the
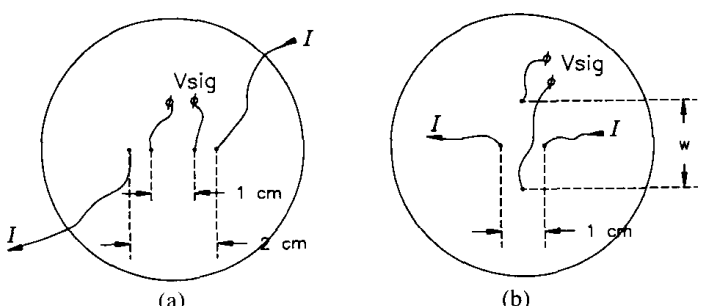

(b)

Fig. 5. (a) A $5-\mathrm{cm}$ diameter Permalloy film $(t=50 \mathrm{~nm})$ with contacts in a magnetoresistor-like geometry. (b) The same film with contacts in a pseudo-Hall-like geometry.

pseudo-Hall device of Fig. 2(b) ( $l>>w$ ). The distortion is extremely small for $w=2$, but it is significant for other values of $w$. This was demonstrated in a circular film of 5-cm diameter and a contact geometry as in Fig. 5(b) with $w=2 \mathrm{~cm}$. A considerable distortion from the sine shape was found, which was caused by the AMR-induced changes in the $\bar{J}$ field [9].

It should be noted that the above calculation provides a complete solution for $\bar{E}^{*}(=-\operatorname{grad} \Phi(\xi, \eta))$ and $\bar{J} *$ ( $=\bar{E} * / \rho^{*}$ ) in the $\xi-\eta$ plane. The solutions for $\bar{J}$ and $\bar{E}$ in the $x-y$ plane can be found with the transformations used in calculation (26).

\section{Other Geometries}

Up till now, some special geometries have been treated. As mentioned previously, the general situation is very difficult to describe. The transformation (25) can be used on a general geometry, leading to an isotropic medium and Laplace equations for $\Psi$ and $\Phi$. However, the boundary equations must also be transformed and an analytical solution can only be expected in very simple structures. With the aid of elaborate computer programs, different geometries can be simulated and compared. This method however is time-consuming and the results are restricted to a limited number of geometries. On the other hand, a few simple rules can easily be deduced from the results of the previous calculations.

In order to be able to compare different types of AMR devices we need a figure of merit, which tells something about the output signal of the device. An important figure, used to optimize the signal-to-noise ratio of a system, is the ratio of signal power $P_{\text {sig }}$ to dissipated power $P_{\text {dis }}$ of a device, when the output signal drives a load of matched resistance. In our case, we want the signal current $I_{\text {sig }}$ to be zero, to eliminate the influence of the contact resistance of the voltage contacts. Furthermore, in many devices a distortion in the signal will occur when $I_{\text {sig }} \neq 0$, because of the magnetization-dependent internal resistance of the device. The signal voltages should be measured with a high-impedance circuit. We can take a high standard resistance for all devices, so that $I_{\text {sig }}$ is effectively zero in every situation, for instance $1 \mathrm{G} \Omega$. The figure of merit $\Xi$ can now be defined as

$$
\Xi=\frac{P_{\mathrm{sig}}}{P_{\mathrm{dis}}}=\frac{\left(\frac{\text { signal voltage swing }}{2}\right)^{2}}{P_{\mathrm{dis}}} \times 10^{-9} .
$$


The load resistance of $10^{9} \Omega$ has the status of a scaling factor. In practice, this factor can be left out and we define

$$
\Xi=\frac{\left(\frac{\text { signal voltage swing }}{2}\right)^{2}}{P_{\mathrm{dis}}} \times\left[\frac{1}{\Omega}\right]
$$

where the multiplication with a dimension $[1 / \Omega]$ is necessary to keep $\boldsymbol{\Xi}$ a dimensionless figure. The ratio of signal power to dissipated power of the device when driving a load of matched resistance is found as

$$
\eta=\frac{\Xi}{R_{\text {in }}}
$$

where $R_{\text {in }}$ is the internal resistance of the device, measured between the voltage contacts (which includes the contact resistance).

In this section, the signal voltage, figure of merit, and internal resistance $R_{\text {in }}$ of a number of devices will be calculated. In all cases the device is driven with a constant current. The contact resistance of all contacts is neglected in the calculations.

For the magnetoresistor of Fig. 2(a) we find

$$
\begin{aligned}
V_{\mathrm{sig}} & =I \frac{\rho}{t} \frac{l}{w}+I \frac{\Delta \rho}{t} \frac{l}{w} \cos (2 \theta) \\
R_{\mathrm{in}} & =\frac{\rho}{t} \frac{l}{w}+\frac{\Delta \rho}{t} \frac{l}{w} \cos (2 \theta) \approx \frac{\rho}{t} \frac{l}{w} \\
\Xi & \approx \frac{\Delta \rho}{\rho} \frac{\Delta \rho}{t} \frac{l}{w} .
\end{aligned}
$$

The internal resistance of the magnetoresistor depends on $\Theta$. For this reason, $\Xi$ can only be calculated approximately. The value of $\Xi$ in (31) is valid for magnetoresistor strips where the voltage contacts are positioned close to the current contacts $\left(l \approx l_{0}\right)$. If this is not the case, a large part of the AMR film is not used effectively and $\Xi$ will have to be adapted for the unused fraction of the film. Calculating $\eta$ for a magnetoresistor with (30a) yields a value $(\Delta \rho / \rho)^{2}$, as expected [1]. For all other devices treated here, $\eta$ has a comparable value.

Three important parameters are apparent in the figure of merit: the length-to-width ratio $l / w$ (a geometrical factor), the relative AMR effect $\Delta \rho / \rho$ (a material parameter), and $\Delta \rho / t$. $\Xi$ can be increased by increasing $l / w$, at the cost of a higher internal resistance of the device. The relative AMR effect can be increased by annealing [5], [10] or by choosing different AMR materials [3]. The factor $\Delta \rho / t$ is inversely proportional to the thickness $t$. As $\Delta \rho$ is approximately independent of $t[16],[17]$, this factor can be increased considerably. However, $\rho$ increases in very thin films, to become infinite when the film becomes electrically discontinuous. In Permalloy this will occur at a film thickness of 5 to $10 \mathrm{~nm}$, depending on the deposition technique. Krongelb [16] has given some results for $\rho$ and $\Delta \rho$ as a function of $t$ for evaporated films. For sputtered films, these values should be comparable

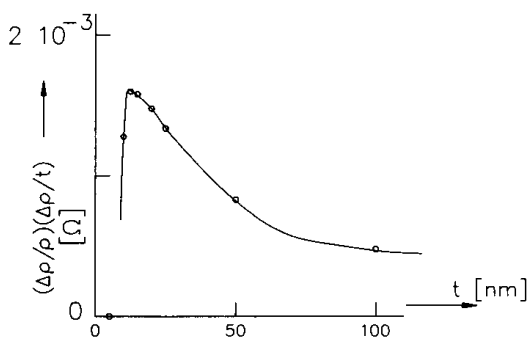

Fig. 6. $(\Delta \rho / \rho)(\Delta \rho / t)$ versus the film thickness $t$ for an evaporated Permalloy film. The plot is based on the results of Krongelb [16].

[17]. In Fig. 6 the product $(\Delta \rho / \rho)(\Delta \rho / t)$ is presented as a function of $t$, based on the results of Krongelb. It is clear, that an optimum film thickness is found around 10 to $20 \mathrm{~nm}$. It should be noted that thinner films tend to be less reproducible. These results of course strongly depend on the deposition technique, the actual AMR material, and possible annealing treatment afterwards. If one is able to produce very thin films with an acceptable resistivity, much can be gained here, providing that the magnetic properties of very thin films are sufficient.

It was concluded in the preceding section, that the voltage contacts of a pseudo-Hall device (Fig. 2(b)) should be small. Furthermore, the current contacts should be far away from the voltage contacts. This implies that $l \gg$ $w$. However, in that case a large portion of the AMR film is not used effectively. This suggests, that an optimum length-to-width ratio exists for pseudo-Hall devices. The effect of the current and voltage contacts on the signal voltage is comparable to that in normal Hall sensors, and is approximated by a correction factor $G(l / w, s / w)$ [7], [13], [18], which describes the influence of the length-towidth ratio and of the width $s$ of the voltage contacts. For small voltage contacts $(s<<w)$, this correction factor reduces to

$$
F\left(\frac{l}{w}\right)=1-\frac{16}{\pi^{2}} e^{-((\pi / 2)(l / w))} .
$$

A plot of $F(l / w)$ versus $l / w$ is given in Fig. 7(a).

The internal resistance of the pseudo-Hall device is hard to calculate, but will be in the order of $\rho / t$, depending on $l / w$ and on the size of the voltage contacts. We find

$$
\begin{gathered}
V_{\mathrm{sig}} \approx I \frac{\Delta \rho}{t} F\left(\frac{l}{w}\right) \sin (2 \theta) \\
R_{\mathrm{in}} \text { in the order of } \frac{\rho}{t} \\
\Xi \approx \frac{\Delta \rho}{\rho} \frac{\Delta \rho}{t} \frac{(F(l / w))^{2}}{l / w} .
\end{gathered}
$$

Fig. 7(b) shows a plot of $(F(l / w))^{2} /(l / w)$ versus $l / w$. A maximum value of just below 0.5 is found for $l / w \approx$ 1.35 .

In general, the optimization of a pseudo-Hall device is quite complicated. If a current $I_{\text {sig }}$ is drawn from the device its internal resistance should be known and the width 


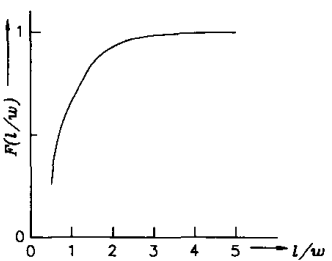

(a)

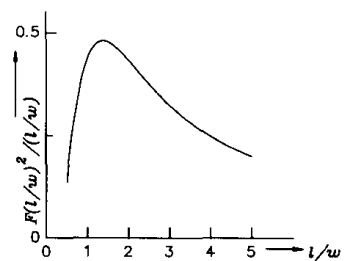

(b)
Fig. 7. (a) The correction factor $F(l / w)$ versus $l / w$ in a pseudo-Hall device with small voltage contacts. (b) $(F(l / w))^{2} /(l / w)$ versus $l / w$ for the same device.

of the voltage contacts should be included in the calculations. One can use approximations, computer simulations [18], or an experimental study [6].

The reduction of the signal voltage for lower values of $l / w$ is caused by the fixed orientation of the electric field near the current contacts. Near the contacts, the AMR effect affects the current density. The influence of the changes in the current density on $V_{\text {sig }}$ is exerted via the resistivity of the material, which is also affected by the AMR effect. As a result, a small distortion in $V_{\text {sig }}$ is introduced. This distortion was observed in measurements on a pseudo-Hall device with $l / w=1$ [9]. It can be concluded, that the correction factor $F(l / w)$ is only approximate.

The value of $\Xi$ for pseudo-Hall devices cannot be increased as easily as in a magnetoresistor, where $\Xi$ is proportional to $l / w$. However, the absence of the large constant term in the signal voltage of a pseudo-Hall device, as it occurs in the magnetoresistor, can be a very useful property.

A signal voltage equivalent to the pseudo-Hall device can be obtained from a magnetoresistor bridge as in Fig. 8 . The four elements in the bridge have length $l$, width $w$, and thickness $t$. The signal voltage is proportional to $\sin (\gamma)$ and is maximum for $\gamma=90^{\circ}$. In that case, we find

$$
\left.\begin{array}{rl}
V_{\text {sig }} & =I \frac{\Delta \rho}{t} \frac{l}{w} \sin (2 \theta) \\
R_{\text {in }} & =\frac{\rho}{t} \frac{l}{w} \\
\Xi & =\frac{\Delta \rho}{\rho} \frac{\Delta \rho}{t} \frac{l}{w} .
\end{array}\right\}
$$

The internal resistance of the bridge is independent of $\theta$. The electrical properties of different bridge structures, such as ring-shaped or meandering devices, can be calculated in the same way.

As a last example, the geometry of Fig. 9 is examined, being a disc-shaped AMR device with four small circular contacts. An approximation of the resistance between two opposite terminals is needed to find the internal resistance of the device. Formula (24) indicates an infinite resistance for point contacts. In the case of circular contacts with a radius $r_{c}$ much less than their mutual distance $w,(24)$ can be used as an approximation of the potential of the con-

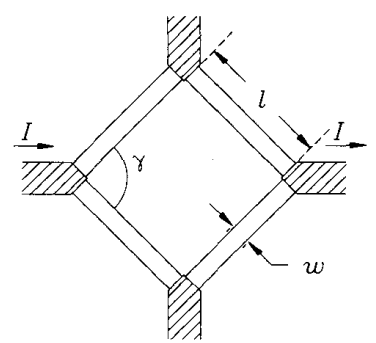

Fig. 8. A magnetoresistor bridge. The four strips have length $l$, width $w$, and thickness $t$. The angle between the strips is $\gamma$.

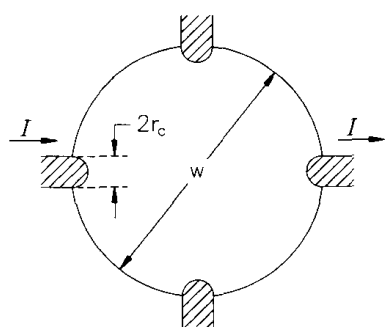

Fig. 9. A disc-shaped AMR device with small circular contacts.

tact. With this, we find that the resistance between two contacts in an infinite film is approximated by

$$
R=\frac{1}{\pi} \frac{\rho}{t} \ln \left(w / r_{c}\right) \quad\left(r_{c} \ll w\right) .
$$

If the contact configuration of Fig. 9 is used in the infinitely wide film, two things become apparent:

- the edge of the film geometry of Fig. 9 will approximately coincide with a current path;

- half of the total current passes between the voltage contacts when the film is infinitely wide.

It can be concluded, that the resistance between two contacts in the device of Fig. 9 is approximated by the resistance between two circular contacts of equal radius in an infinitely wide film, if it is multiplied by two. For the signal voltage it can be concluded, that (29a), multiplied by two, can be used as an approximation. We find

$$
\left.\begin{array}{rlr}
V_{\mathrm{sig}} & =\frac{2}{\pi} I \frac{\Delta \rho}{t} \sin (2 \theta) & \\
R_{\mathrm{in}} & =\frac{2}{\pi} \frac{\rho}{t} \ln \left(\frac{w}{r_{c}^{v}}\right) & \left(r_{c}^{v} \ll w\right) \\
\Xi & =\frac{2}{\pi} \frac{\Delta \rho}{\rho} \frac{\Delta \rho}{t} \frac{1}{\ln \left(w / r_{c}^{c}\right)} & \left(r_{c}^{c} \ll w\right)
\end{array}\right\}(35)
$$

with $r_{c}^{v}$ and $r_{c}^{c}$ representing the radii of the voltage contacts and current contacts, respectively. In general, these radii are not necessarily equal.

To have an idea of the value of $R_{\text {in }}$ and $\Xi$ for an actual device, we take a contact radius of $25 \mu \mathrm{m}$ and a device diameter of $2 \mathrm{~mm}$. The factor $\ln \left(w / r_{c}\right)$ is found to be approximately 4.4. In experiments [9] a somewhat higher signal voltage amplitude is found. This is probably due to 
the effect of the film edge on the current density of the film, which is neglected in (35).

The choice of the geometry of an AMR device usually depends on more requirements than the figure of merit $\Xi$ and the signal voltage amplitude. For instance, the internal resistance of the device, the signal-to-noise ratio, the maximum permittable current density at any point in the film $\left(J_{\max } \approx 10^{10} \mathrm{~A} / \mathrm{m}^{2}\right.$ for Permalloy $)$, the geometry of the device or its magnetic properties can be important parameters. Finally, the device should have a good thermal and long-term stability. These properties can sometimes be improved by changing the device geometry. As an example, consider a device where the stability of the relatively large current contact limits its thermal stability due to inhomogeneous changes of the contact properties throughout the contact area. In such a device, the influence of these contact boundaries should be reduced by positioning the current contacts further away, or by using smaller contacts, thus increasing the influence of the stable film boundaries.

\section{ConClusions}

In this paper the AMR effect in an AMR thin film with homogeneous magnetization is investigated. The differential equations for the potential $\Phi$ in the film and for the vector field $\bar{\Psi}$, which is related to the current density in the film, together with their boundary equations are given.

It is concluded, that in a practical situation an AMR device should be operated by four-point measurement. To obtain good stability, it should be driven by a constant current, while the signal voltage should be measured with high impedance. Furthermore, the current density should be independent of the AMR effect to obtain an undistorted signal voltage with maximum amplitude.

A homogeneous current density in an infinitely wide thin film is not affected by the AMR effect. Concerning the boundaries of an AMR thin film, one can conclude, that a film edge forces the current density to have a fixed orientation, while the highly conductive voltage or current contacts provide a fixed electric field. The influence of contacts on the current density should be kept small, especially in the case of a pseudo-Hall element.

The problem of four point contacts in an infinitely wide AMR thin film can be solved analytically with the aid of a special transformation. The results are applicable as an approximation of the behavior of a disc-shaped AMR device.

A figure of merit $\Xi$ is defined that can be used to compare different AMR devices. This figure is calculated for a number of devices, together with the signal voltage and the internal resistance of the device.

It can be concluded, that an AMR thin film used in AMR devices, where the signal voltage is measured with high impedance, has an optimum film thickness. The optimum thickness depends on the material, the deposition technique, and possible annealing steps. For evaporated Permalloy films, an optimum film thickness is found between 10 and $20 \mathrm{~nm}$. Furthermore, a pseudo-Hall element has an optimum length-to-width ratio of about 1.35 .
The final choice of the AMR device in a special application will depend on other requirements such as the internal resistance and signal-to-noise ratio, magnetic properties, occupied space, and technological realization.

\section{REFERENCES}

[1] D. A. Thompson, L. T. Romankiw, and A. F. Mayadas, "Thin film magnetoresistors in memory, storage, and related applications, "IEEE Trans. Magn., vol. MAG-11, pp. 1039-1050, July 1975.

[2] K. J. M. Eijkel, P. Groot, J. W. Wieberdink, H. Leeuwis, J. H. J. Fluitman, and Th. J. A. Popma, "A thin-film magnetoresistive angle detector," in print in Sensors and Actuators, 1989.

[3] T. R. McGuire and R. I. Potter, "Anisotropic magnetoresistance in ferromagnetic 3d alloys,"' IEEE Trans. Magn., vol. MAG-11, pp. 1018-1037, July 1975.

[4] I. L. Sanders, "NiFeCo, an alternative to Permalloy for bubble domain detection," IEEE Trans. Magn., vol. MAG-19, pp. 104-110, Mar. 1983.

[5] S. Krongelb, A. Gangulee, and G. Das, "Annealing of thin magnetoresistive Permalloy films," IEEE Trans. Magn., vol. MAG-9, pp. 568-570, Sept. 1973

[6] C. P. Battarel and M. Galinier, "Optimization of the planar Hall effect in ferromagnetic thin films for device design," IEEE Trans. Magn., vol. MAG-5, pp. 18-22, Mar. 1969.

[7] J. Haeusler, “Die Geometriefunktion vierelektrodiger Hallgeneratoren," Arch. Elektrotech., vol. 52, pp. 11-19, 1968.

[8] J. Lenz, K. Kawai, R. Fryer, and L. Strandjord, "Comparison of five readout techniques for an integrated magnetometer," presented at the 1986 IEEE Solid-State Sensor and Actuator Workshop.

[9] K. J. M. Eijkel, “A thin-film magnetoresistive angle detector," Ph.D. dissertation, University of Twente, Enschede, The Netherlands, 1988

[10] A. D. Butherus and S. Nakahara, "Annealing kinetics of thin Permalloy films," IEEE Trans. Magn., vol. MAG-21, pp. 1301-1305, July 1985.

[11] R. L. Coren and H. J. Juretschke, "Magnetoresistance and magnetic switching in Permalloy films," J. Appl. Phys., vol. 32, pp. 292S293S, Mar. 1961

[12] R. F. Soohoo, Magnetic Thin Films. New York, NY: Harper \& Row, 1965.

[13] R. M. de Ridder, "Thin-film magnetoresistive magnetometer," Ph.D. dissertation, University of Twente, Enschede, The Netherlands, 1988.

[14] U. Dibbern, "Sensors based on the magnetoresistive effect," Sensors and Actuators, vol. 4, pp. 221-227, 1983.

[15] H. Th. Hildering, "Het geleidingsvermogen van polykristallijn materiaal," University of Twente, Int. Rep. UT/EL/TDM 1228.1821, 1974.

[16] S. Krongelb, "The preparation and properties of magnetoresistive Permalloy films," J. Electron. Mat., vol. 2, pp. 227-238, 1973.

[17] K. Solt, "Magnetic, structural and magnetoresistive properties of magnetron-sputtered thin NiFe films,"' Thin Solid Films, vol. 125, pp. $251-256,1985$.

[18] H. Leeuwis, "Effect of 'overlapping' voltage contacts in planar Hall transducers," Sensors and Actuators, vol. 4, pp. 17-24, 1983.

Kees J. M. Eijkel was born in Heiloo, The Netherlands, on October 1, 1959. He received the M.Sc. degree in mathematics from the University of Amsterdam, Amsterdam, The Netherlands, in 1983 and the Ph.D. degree from the University of Twente, Enschede, The Netherlands, in 1988.

Jan H. Fluitman was born in Beverwijk, The Netherlands, on March 23, 1938. He received the M.Sc. and Ph.D. degrees in physics from the University of Amsterdam, Amsterdam, The Netherlands, in 1966 and 1970 , respectively.

He joined the University of Twente, Enschede, The Netherlands, in 1970 , employed to teach applied magnetism and conducting research in the field of magnetic recording and magnetic transducers. Since 1982 he has been Full Professor of Transducer Science. His interests now are in magnetic, optic, and micromechanic transduction principles. 